\title{
La représentation du dialecte anglo-gallois dans le roman social de Lewis Jones intitulé Cwmardy (1937)
}

The representation of the Anglo-Welsh dialect in Lewis Jones's social novel Cwmardy (1937)

\section{Gaëlle Le Corre}

\section{OpenEdition \\ Journals}

Édition électronique

URL : https://journals.openedition.org/lbl/305

DOl : $10.4000 / / \mathrm{lbl} .305$

ISSN : $2727-9383$

\section{Éditeur}

Université de Bretagne Occidentale - UBO

\section{Édition imprimée}

Date de publication : 1 mai 2017

Pagination : 7-28

ISBN : $979-10-92331-31-8$

ISSN : $1270-2412$

\section{Référence électronique}

Gaëlle Le Corre, «La représentation du dialecte anglo-gallois dans le roman social de Lewis Jones intitulé Cwmardy (1937) », La Bretagne Linguistique [En ligne], 21 | 2017, mis en ligne le 01 mai 2020, consulté le 26 mars 2022. URL : http://journals.openedition.org/lbl/305; DOI : https://doi.org/ $10.4000 / \mathrm{lbl} .305$

\section{(c) (i)}

La Bretagne Linguistique est mise à disposition selon les termes de la Licence Creative Commons Attribution 4.0 International. 


\section{La représentation du dialecte anglo-gallois dans le roman social de Lewis Jones intitulé Cwmardy (1937)}

Le pays de Galles connut un essor économique sans précédent durant la période d'industrialisation massive de la Grande-Bretagne. Ce fut principalement le sud-est du pays de Galles qui profita de cette croissance économique, où la production de fer, de charbon et d'acier était l'une des plus prolifiques du monde. La vallée de la Rhondda, grâce à ses mines de charbon, fut probablement la région la plus touchée par ces changements économiques et sociaux ${ }^{1}$.

Un tel développement industriel eut une incidence considérable sur le mode de vie et la culture de la région. En effet, cette période fut marquée par l'influence grandissante de la langue anglaise qui commença à supplanter peu à peu le gallois, langue qui était alors très largement dominante. Bien que les premières vagues migratoires fussent internes au pays de Galles, elles furent supplantées par une migration extérieure, principalement originaire du sud-ouest de l'Angleterre ${ }^{2}$.

* Maitre de conférences d'anglais, UBO/CBRC (EA 4451), gaelle.lecorre@univ-brest.fr

1. Kenneth S. HopkIns, Rhondda Past and Future, Ferndale, 1974 ; Evan D. LEWIS, Rhondda Valleys: a Study in Industrial Development: 1800 to the Present Day, London, Phoenix House, 1959.

2. E. D. LEWIS, « Population Changes and Social Life », dans K. S. Hopkins (dir.), Rhondda Past and Future, op. cit., p. 239. 
L'anglicisation rapide de la région, ainsi que les influences dialectales externes et internes au pays, ont ainsi donné naissance au vernaculaire anglo-gallois.

Toutes ces évolutions inspirèrent de nombreux écrivains, tels que Jack Jones ou Richard Llewllyn, qui ressentirent le besoin d'exprimer leur nostalgie face à ce pays qu'ils ne reconnaissaient plus. Lewis Jones écrivit, entre 1937 et 1939, deux romans sociaux intitulés Cwmardy et We Live, dans lesquels il narra la vie, les déboires ainsi que l'engagement politique d'une famille de mineurs de la Rhondda.

Malgré l'engagement politique de ses œuvres, Jones dépeignit avec justesse le milieu des mineurs entre les années 1890 et 1930. Cwmardy se déroule entre 1890-1914 tandis que We Live décrit la dépression économique des années 1920-1930. Jones est un des rares auteurs à avoir tenté de représenter le parler dialectal des mineurs de l'époque. Ce choix stylistique permet d'ancrer pleinement ses personnages dans la réalité sociale, économique et linguistique de la région.

À première vue, Lewis Jones donne l'impression de représenter fidèlement le vernaculaire de la population ouvrière de l'époque. Cependant, une étude détaillée de certaines variations, notamment l'emploi du be invariant et des formes périphrastiques be $+V$-ing et $d o$, nous invite à la prudence. Ainsi, l'analyse de ces variations semble indiquer que le langage dialectal des personnages principaux du roman Cwmardy n'est peut-être pas aussi réaliste que le contexte sociohistorique qu'il dépeint. Pour tenter de répondre à cette question, ces variables seront analysées quantitativement mais aussi qualitativement. Afin d'obtenir des éléments de comparaison pertinents, ces données seront comparées à d'autres travaux de recherche consacrés au vernaculaire anglo-gallois, à savoir le Survey of Anglo Welsh Dialect $(\mathrm{SAWD})^{3}$ mais aussi à la recherche doctorale de Ceri George ${ }^{4}$ dont une partie englobe le dialecte de Vallée de la Rhondda. Notre analyse comparative sera également inspirée des données obtenues suite à

3. David R. PARrY, The Survey of Anglo-Welsh Dialects. Volume 1: The South-East, Swansea, publication privée de l'éditeur, 1977 ; D. R. PARrY, The Survey of Anglo-Welsh Dialects. Volume 2: The South-West, Swansea, publication privée de l'éditeur, 1979.

4. Ceri George, Community and Coal: An Investigation of the English-Language Dialect of the Rhondda Valley, Mid Glamorgan, Swansea, PhD University of Swansea, 1990. 
l'écoute de témoignages de mineurs enregistrés en 1972 et 1974, dans le cadre de la South Wales Coalfield Collection ${ }^{5}$.

Localisation de la vallée de la Rhondda au pays de Galles ${ }^{6}$

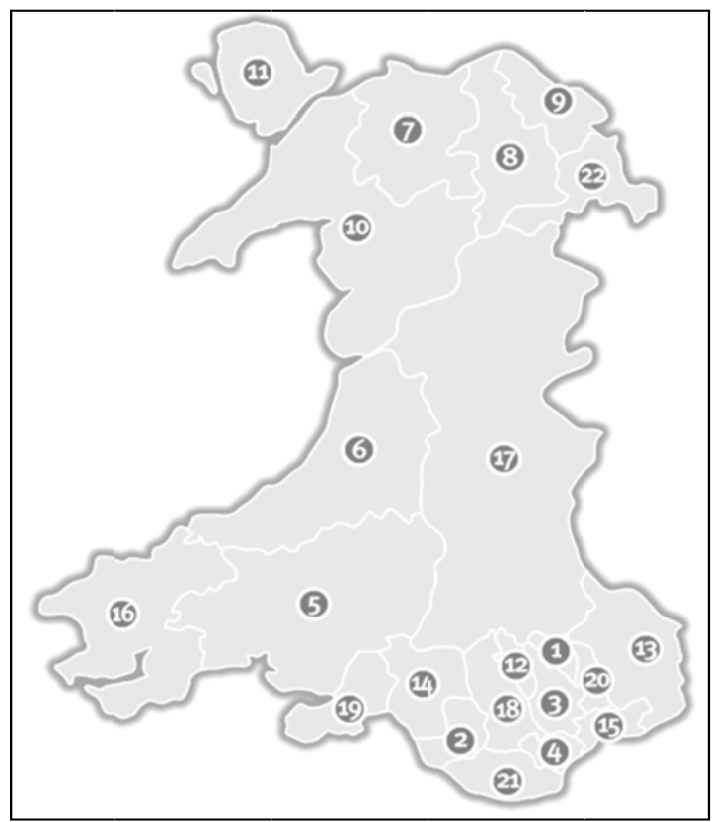

1. Blaenau Gwent

2. Bridgend

3. Caerphilly

4. Cardiff

5. Carmarthenshire

6. Ceredigion

7. Conwy

8. Denbighshire

9. Flintshire

10. Gwynedd

11. Isle of Anglesey

12. Merthyr Tydfil

13. Monmouthshire

14. Neath Port Talbot

15. Newport

16. Pembrokeshire

17. Powys

18. Rhondda Cynon Taff

19. Swansea

20. Torfaen

21. Vale of Glamorgan

22. Wrexham

\section{Cwmardy : métaphore de la vallée de la Rhondda}

\section{Un enfant du pays}

Lewis Jones naquit en 1897 à Clydach Vale, dans la vallée de la Rhondda. Il commença à travailler dans les mines de Cumbrian dès l'âge de douze ans. Son expérience professionnelle et son enfance dans cette région ont inspiré les évènements ainsi que les personnages dépeints

5. The South West Coalfield History Project, The South Wales Miners' Library, Swansea University, 1971-1974, 1979-1982. Documents audio n 164, 165, 168, 169, 180, 181, 313, 365.

6. Source : Stay in Wales, 2016, [en ligne], consulté le 12 mars 2016, disponible sur : [https://www.stayinwales.co.uk/wales.cfm?counties]. 
dans ses deux romans ${ }^{7}$. Les émeutes décrites dans les chapitres 8, 9 et 10 font directement référence aux révoltes de Tonypandy qui se déroulèrent en 1910. Il s'engagea très tôt pour la cause des mineurs. Avant la Première Guerre mondiale, il devint le plus jeune président de la section syndicale de Cumbrian. Grâce à une bourse offerte par le syndicat, il étudia au Central Labour College de Londres, entre 1923 et 1925, où il rencontra Jack Jones qui l'inspira grandement dans l'élaboration de son personnage principal, Len. Membre du parti communiste, Lewis Jones se voua corps et âme à son engagement politique ainsi qu'à la défense de la cause des mineurs.

Il y a donc une adéquation patente entre la réalité politique et sociale de l'époque et les évènements décrits dans l'œuvre. Ce réalisme se manifeste également dans les dialogues, puisque nous observons une dichotomie linguistique très nette entre le langage des ouvriers et celui des classes supérieures. Cette frontière sociale est donc représentée linguistiquement par l'emploi de certaines caractéristiques langagières propres au registre non standard mais aussi au vernaculaire anglogallois.

\section{L'idiolecte des personnages}

Nous recensons de nombreux lexèmes caractéristiques du dialecte local, la plupart étant directement empruntés du gallois : bach (petit), twp (stupid), muniferni (grand dieu), mamgu (grand-mère). D'autres sont directement associés au lexique propre à la communauté des mineurs : black legs or scabs (mineurs non grévistes), a journey (ensemble de 9 ou 10 wagons). Nous retrouvons également des constructions non standard communes à de nombreuses variétés de l'anglais telles que les doubles négations (don't cry no more), les prétérits et participes passés irréguliers (knowed, broked), l'emploi d'un adjectif à la place d'un adverbe (we want to see you very particular). Jones eut également recours à des structures caractéristiques du vernaculaire anglo-gallois telles que la thématisation du prédicat (fine man you be) et la généralisation du tag is it (in't you satisfied).

7. Hywel FranCIS, «Foreword», dans L. Jones, Cwmardy, We Live, Camarthen, Parthian, The Library of Wales, 2006, p. IX-XIV. 
La présente étude se base uniquement sur les dialogues extraits de l'œuvre car ces derniers sont les seuls à fournir des instances non standard. Cependant, tous les personnages ne possèdent pas un discours dialectalement marqué. En effet, la différence de statut social ou de niveau d'instruction des divers personnages est clairement signifiée par leurs idiolectes. Le personnage Lord Cwmardy (propriétaire des mines), emploie un langage standard tout au long du roman. Le vernaculaire anglo-gallois est donc est un marqueur à la fois identitaire et social. Deux registres sont ainsi clairement mis en opposition dans le roman : le registre paritaire, celui de la communauté, opposé au registre disparitaire, principalement maitrisé par les élites. Ces termes sont ainsi définis par les linguistes Jean Le Dû et Yves Le Berre ${ }^{8}$ :

«Le registre "paritaire" [...] identifie le locuteur comme appartenant à sa région. Il est alors représenté par une langue régionale [...] d'autre part il identifie le locuteur comme appartenant à une classe sociale (ouvrier, paysan, artisan d'un côté, bourgeois, aristocrate, intellectuel de l'autre). [...][L]e registre de la disparité est toujours celui des relations qui s'établissent dans la dimension verticale de la société (supérieurs/inférieurs); le registre de la parité est réservé aux communications établies dans la dimension horizontale (entre égaux, semblables, intimes).»

L'usage du vernaculaire non standard des personnages est un moyen de les ancrer socialement et de signifier l'appartenance à la communauté et aux valeurs qui lui sont associées. Cette forme langagière jouit donc, elle aussi, d'une forme de prestige, plus discrète mais partagée par l'ensemble des mineurs ${ }^{9}$. La maitrise de la langue standard n'est cependant pas l'apanage des classes dominantes. En effet, les person-

8. Jean LE DÛ, Yves LE BERRE, «Le double jeu de la langue », dans J.-M. Eloy (dir.), La qualité de la langue? Le cas du français, Paris, Champion, 1995, p. 251-268.

9. Labov distingue le « overt prestige » du " covert prestige ». Le « overt prestige » renvoie à la langue normée et standard à laquelle les locuteurs cherchent à s'identifier (William LABOV, The Social Stratification of English in New York. City, Washington, DC, Center for Applied Linguistics, 1966). Le « covert prestige » associe l'emploi de formes non-standard comme l'expression linguistique d'une appartenance à une communauté. Ces formes sont naturellement associées à des qualités favorables telles que la loyauté ou l'amitié (Peter Trudgill, A Glossary of sociolinguistics, Oxford, Oxford University Press, 2003, p. 30). 
nages instruits, ou jouant un rôle politique important, emploient généralement un registre standard ; c'est le cas par exemple du syndicaliste Ezra Jones et de sa fille, Mary. Ainsi, le niveau d'éducation des personnages, ou tout du moins de leur conscience politique, est également exprimé linguistiquement par la maîtrise des codes linguistiques de la classe dominante.

À travers l'étude de trois variations non standard, à savoir be invariant et les constructions périphrastiques do et be + Ving, nous tenterons de déterminer si la représentation du vernaculaire local est aussi fidèle que la réalité sociale dépeinte dans l’œuvre.

\section{Be Invariant}

\section{Valeur Historique $B e$ Invariant}

En vieil-anglais, deux conjugaisons du verbe être au présent coexistaient : wesan et bēon. Ainsi, le vieil-anglais distingue les formes en $B$ - en en $S$-. La valeur aspectuelle des formes en $B$ - servait à décrire des états ou des actions considérés comme habituels, génériques ou atemporels. Bēon pouvait parfois également être employé pour décrire des procès futurs ${ }^{10}$.

Les formes en $S$ - représentaient des procès passés ou présents singuliers, non habituels et ponctuels ${ }^{11} \mathrm{La}$ forme wesan pouvait cependant également décrire des procès à valeur atemporelle.

La convergence de ces valeurs permet ainsi de mieux comprendre pourquoi ces formes se sont par la suite combinées pour ne former qu'un seul paradigme du verbe être ${ }^{12}$. La coexistence de ces deux formes est également caractéristique des langues celtiques et principalement brittoniques. Bien que cette distinction ait aujourd'hui disparu en anglais standard, elle peut être encore observée dans le vernaculaire du sud-ouest de l'Angleterre ainsi que dans le dialecte anglo-gallois. Il est

10. Elizabeth TraugotT, «Syntax », dans R. M. Hogg (dir.), The Cambridge History of the English Language, Volume 1: The Beginnings to 1066, Cambridge, Cambridge University Press, 1992, p. 178.

11. André CRÉPIN, Deux mille ans de langue anglaise, Paris, Nathan, 1994, p. 86.

12. Gary GERMAN, «Du brittonique à l'anglais africain-américain vernaculaire (AAAV) : l'étrange odyssée de be et do périphrastiques habituels », dans L.-E. Edlund, A.Van Nahl (dir.), Journal of Dialectology and Geolinguistics, Bamburg, Mouton de Gruyters, 2006, p. 12-62. 
donc fort possible que, sur ce point, le brittonique ait eu une influence sur le vieil-anglais ainsi que sur les vernaculaires anglo-gallois et du sudouest de l'Angleterre ${ }^{13}$. En effet, dans les vernaculaires non-standard, be invariant est utilisé pour décrire des habitudes dans le présent et possède parfois une visée prospective :

«I be in the dark of the pit all day. » (Big Jim, chapitre 1)

[«Je passe mes journées dans le noir au fond de la mine. »]

"Let us hope he 'ont forget his poor old mam and dad when he be up in the world.» (Shane, chapitre 2)

[«Espérons qu’il n’oublie pas sa pauvre maman et son papa quand il se fera une place dans la société. »]

\section{Be Invariant dans Cwmardy}

Afin d'analyser de quelles manières Lewis Jones emploie la copule be sous sa forme non fléchie, il convient tout d'abord de référencer l'ensemble des instances employées dans l'œuvre et d'analyser ensuite les contextes discursifs ainsi que les valeurs aspectuelles qui lui sont associés. Ces données seront ensuite comparées à d'autres analyses sociolinguistiques.

Len

Len, personnage principal du roman, emploie des formes invariantes uniquement dans la première partie du roman. Il abandonne peu à peu le vernaculaire non standard à mesure que croît son engagement politique. Il y a donc une rupture nette dans son idiolecte entre la première et la deuxième partie du roman. Sa prise de conscience politique est donc représentée linguistiquement par l'abandon progressif des formes non standard, et dans le cas présent, des formes invariantes. Len devient alors l'emblème du mineur autodidacte et idéaliste œuvrant pour le bien commun et l'amélioration de la condition prolétarienne. Paradoxalement, l'identification à la cause des mineurs est marquée par une rupture linguistique et une distanciation par rapport à leurs codes langagiers. Coupland et Thomas ${ }^{14}$ ont cependant montré que les

13. G. GERMAN, ibidem.

14. Nikolas Coupland, Alan R. Thomas, English in Wales: Diversity, Conflict and Change, Clevedon, Philadelphia, Multilingual matters LTD, 1990. 
hommes politiques gallois membres du parti travailliste (Neil Kinnock, Denzil Davies et Barry Jones) avaient tendance à conserver un accent gallois plus marqué que leurs homologues conservateurs. Au regard de cette étude, il semble donc critiquable que Jones ait choisi de standardiser de manière aussi radicale l'idiolecte de Len.

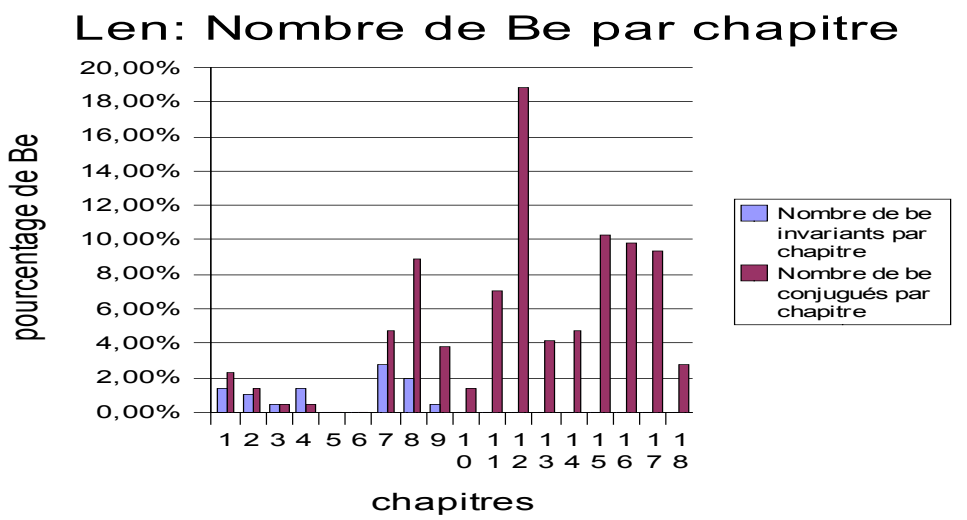

Big Jim

Nous comptabilisons 92 formes invariantes contre 112 fléchies, certaines formes fléchies étant cependant non standard (ex. Many is the time). Ces variables sont réparties de la manière suivante dans l'œuvre:

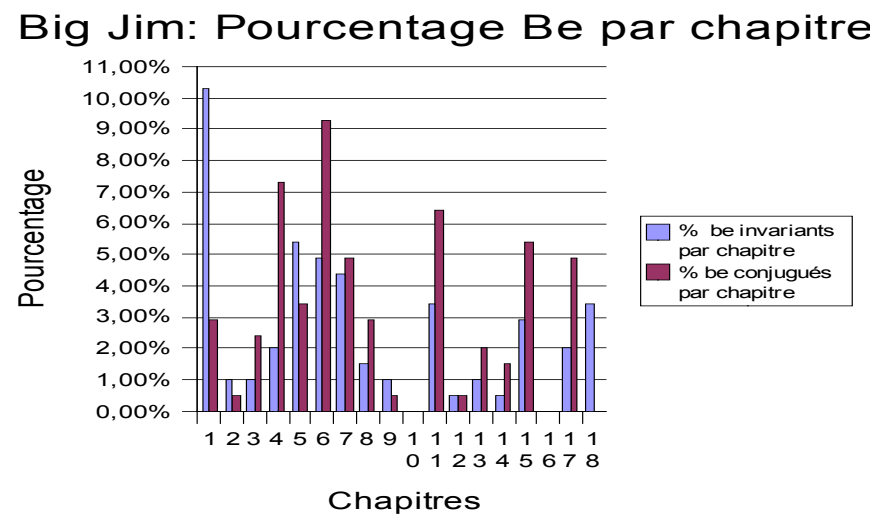

Le manque de constance est assez prégnant. En effet, seuls cinq chapitres contiennent majoritairement des formes invariantes, à savoir 
les chapitres 1, 2, 5, 9 et 18. Cette préférence est d'ailleurs fortement marquée dans le premier et le dernier chapitre. Dans les cinq chapitres précédemment cités, Big Jim s'adresse toujours à des personnes qui lui sont proches : son épouse, ses enfants ou camarades mineurs. Les formes invariantes sont donc associées à des valeurs émotionnelles fortes. Dans le chapitre introductif, Big Jim est en haut de la vallée avec son jeune fils Len, ce moment privilégié est donc propice à l'emploi d'un registre paritaire.

" "Come," "it be getting late and your mam will be wondering where we be." [...] "That be good, muniferni. When I do tell your mam what you have said her belly will shake with laughing. Ho-bo-bo!" [...]

"Ay, I think they all be there, boy bach," "But," "you did ought to see it first thing in the morning when the sun be rising. Ah, that be a sight for sore eyes." " (Chapitre 1)

[" "Viens. Il se fait tard et ta mère va se demander où on est. [...]

En voilà une bonne, mon Dieu. Quand je vais raconter à ta mère ce que tu m'as dit, elle va se tordre de rire. Ho-ho-ho. "[...]

"Ouais, je pense qu'ils sont tous là, mon petit garçon. Mais tu aurais dû voir comment c'était dès le matin au lever du soleil. Ah, c'était un vrai plaisir pour les yeux.” »]

Les formes fléchies sont quant à elles principalement employées dans des contextes où le personnage établit une distanciation très marquée entre lui et son interlocuteur, comme nous pouvons le voir dans l'exemple suivant dans lequel Big Jim s'adresse avec véhémence à Evan the Overman qui refuse d'admettre que son fils est le futur père de l'enfant que porte Jane, la fille de Big Jim. Tout au long de la scène, son langage ne contient aucune forme invariante :

"'You say your father is an official. Yes that is true. But you don't tell us how he came to be one. Answer that, Evan bach, before you open your mouth again. But, there, perbaps you don't know." [...]

"That is one thing you will never see. I am sorry for you. The son of such a man as your father is not fit to enter the family of Jim the Big. I would sooner find the father of my gel's baby in the gutter than in this house." (Chapitre 4)

[ " "Tu dis que ton père un officiel. Oui c'est vrai. Mais tu ne nous dis pas comment il y est parvenu. Réponds à ça, Petit Evan, avant d'ouvrir à nouveau la bouche. Ah mais oui, peut-être que tu ne le sais pas. [...]" 
"C’est une chose que tu ne verras jamais. Je suis désolé pour toi. Le fils d'un homme tel que ton père n'est pas digne d'entrer dans la famille du Grand Jim. Je préférerais voir le père du bébé de mon petit ange dans le caniveau plutôt que dans cette maison." »]

Il y a donc, dans le discours de Big Jim, une dichotomie très nette entre les contextes d'emploi de be invariant et des formes fléchies. La forme non fléchie est associée à un langage paritaire tandis que les constructions fléchies servent à marquer une forte distance entre l'énonciateur et le co-énonciateur. Lewis Jones a donc choisi de dépeindre un personnage qui adapte son discours non seulement en fonction du public auquel il s'adresse mais aussi en fonction de l'impression qu'il veut donner à son interlocuteur - stratégie qui n'est pas partagée par les deux autres personnages principaux.

\section{Shane}

À nouveau, nous observons une dichotomie très marquée dans l'emploi des formes invariantes entre la première et la deuxième partie de l'œuvre :

\section{Shane: Pourcentage Be par chapitre}

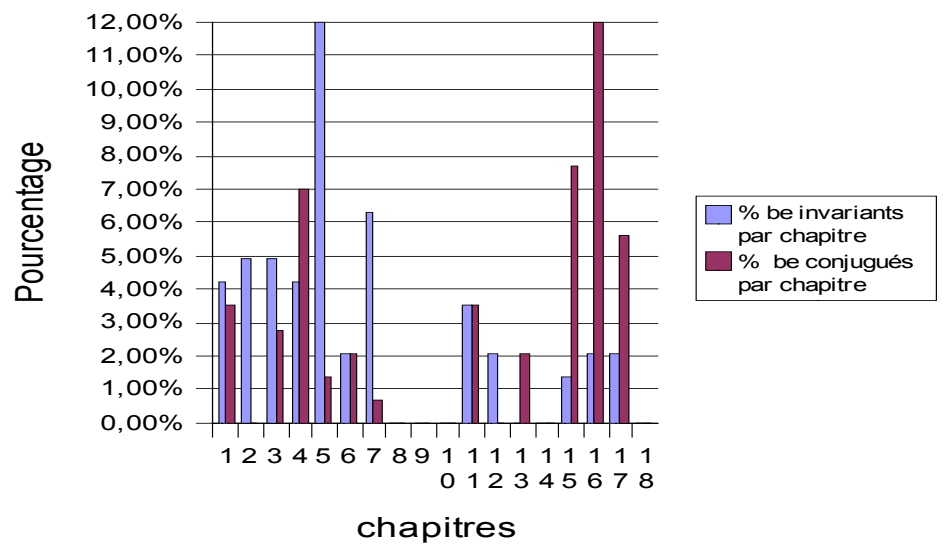

Les contextes énonciatifs dans lesquels les formes invariantes sont employées diffèrent sensiblement de ceux employés par Big Jim. En effet, Shane ne semble pas adapter son langage en fonction de son interlocuteur, comme nous pouvons le constater dans le chapitre 2 où 
elle a une violente altercation avec l'instituteur de Len, qui affirme que son fils fait l'école buissonnière. Le contexte est clairement disparitaire et l'énonciateur est en conflit ouvert avec le co-énonciateur. Or dans ce contexte, Shane emploie uniquement des formes invariantes:

«Lose school?

"Who do say my boy lose school? Why, he be here every day like a dock ever since he started, Huh! Be very careful what you do say, my man! 'Member there be still a law, even for the poor." "I don't believe my boy 'ood do such a thing," "I 'oodn't believe no man, not even if he went down on his bended knees before my eyes [...]." »

[«L'école buissonnière?

"Qui dit que mon fils fait l'école buissonnière ?" Pourquoi, il y est tous les jours depuis qu'il a commencé, hein! Fais bien attention à ce que tu dis! Rappelle-toi qu'il y a une justice, même pour les pauvres. Je ne peux pas croire que mon fils ferait une chose pareille. Je ne croirais personne même s'il s'agenouillait devant moi [...].»]

D'un point de vue sociolinguistique, un tel choix semble paradoxal car les femmes issues des classes populaires sont plus enclines que leurs homologues masculins à adopter les codes linguistiques des classes sociales plus élevées ${ }^{15}$. Il convient néanmoins d'admettre qu'à cette époque, les femmes de mineurs vivaient en cercle fermé et côtoyaient très rarement une population qui n'appartenait pas à leur milieu social. Cette hypothèse paraît cependant caduque lorsqu'elle est mise en perspective avec la rupture brutale observée dans la deuxième partie de l'œuvre. En effet, l'environnement social dans lequel évolue Shane reste le même tout au long de l'œuvre.

Cette standardisation relativement soudaine parait donc peu probable. Il y a donc des failles flagrantes dans le système linguistique employé par Lewis Jones puisque nous constatons une rupture très nette dans l'idiolecte des personnages entre la première et la deuxième partie du roman. Le cas de Len est très révélateur. Bien qu'il se soit quelque peu instruit au cours du roman, grâce à la lecture d'ouvrages recommandés par son mentor Ezra Jones, il n'a cependant aucunement

15. Peter TRudgILl, The Social Differentiation of English in Norwich, Cambridge, Cambridge University Press, 1974, p. 94. 
quitté son milieu d'origine, ce qui aurait pu ainsi expliquer cette rupture brutale. À partir du chapitre 9, qui retrace les émeutes de Tonypandy de 1910, Len modifie soudainement son langage, qui se standardise en moins de trois chapitres. Or, ces derniers couvrent le même évènement. Il n'y a donc aucune rupture chronologique qui puisse justifier d'un point de vue linguistique un changement aussi drastique.

De plus, nous observons une incohérence dans les codes énonciatifs entre les personnages, d'autant plus que les variables non standard sont employées dans un contexte disparitaire et sont plus fréquemment associées à des locuteurs féminins, ce qui semble linguistiquement peu vraisemblable ${ }^{16}$.

\section{Valeurs des formes non-standard}

Les formes invariantes sont employées au présent dans l'œuvre, ce qui est en adéquation avec la réalité linguistique de l'époque puisque la forme en $B$ - n'est jamais usitée au passé en vieil-anglais. L'analyse des valeurs des formes non fléchies dans le roman offre cependant des résultats plus contrastés. Certaines variables non fléchies sont employées pour décrire des vérités générales, des procès habituels ou atemporels, ce qui semble en adéquation avec les valeurs intrinsèques de la forme en $B$ - :

"I be in the dark of the pit all day. " (Big Jim, chapitre 1)

[« Je suis dans l'obscurité de la mine toute la journée. »]

"There be nothing fair in this world.» (Shane, chapitre 4)

[« Il n'y a rien de juste dans ce monde.»]

La plupart des formes invariantes employées par l'auteur décrivent fréquemment un état spécifique du sujet, l'ancrage temporel est donc le moment présent :

"Your father be in an anful state all of a sudden." (Shane, chapitre 3)

["Ton père est dans un sale état tout d'un coup. »]

«Well, boy bach, you be a big man to-day, starting school, eh?» (Big Jim, chapitre 2) [«Et bien mon p'tit gars, tu es un vrai homme aujourd'hui, on commence l'école hein?»] 
Be invariant peut donc tout aussi bien être employé pour décrire des procès ponctuels et/ou itératifs. La plupart des formes invariantes usitées par les locuteurs interrogés dans le South West Coalfield History Project font référence à des procès duratifs ou itératifs :

«There be no traffic on Sunday like. » (AUD 164)

[ "Il n'y a aucune circulation le dimanche. »]

"It be the two on the top shelf. » (AUD 180)

[« Ce sont les deux sur l'étagère du haut. »]

Ces enregistrements contiennent néanmoins des formes invariantes employées pour décrire des procès ponctuels. Les choix linguistiques de l'auteur à ce sujet ne sont donc pas complètement erronés. En effet, bien que la valeur d'origine des formes en B- serve à représenter des procès habituels, génériques, atemporels et futurs, la valeur aspectuelle de be demeure assez fluctuante dans les variétés d'anglais non standard employant cette variable ${ }^{17}$.

À plusieurs reprises, Lewis Jones a recours à la forme invariante dans des constructions négatives. Or ces dernières ne sont aucunement répertoriées dans le Survey of Anglo-Welsh Dialect (SAWD) ${ }^{18}$ et le Survey of English Dialect (SED) ${ }^{19}$ qui recensent les variables non standard bain't, ain't et inna à la première personne du singulier et à la troisième personne du pluriel et du singulier. La réalité linguistique de l'époque était donc bien plus diversifiée. Be invariant est quasiment la seule variable non standard employée au présent dans les énoncés négatifs dans le roman; la variable ain't étant recensée à une seule reprise. Bien que les diverses valeurs aspectuelles de be invariant dans le roman paraissent idiomatiques, Lewis Jones semble avoir simplifié le vernaculaire anglogallois en choisissant d'employer uniquement la variable be non fléchie. Il y a donc non seulement une standardisation du dialecte mais aussi une simplification de ce dernier.

17. TRISTAM, communication personnelle, citée dans G. GERMAN, op. cit., p. 13

18. D. PARRY, op. cit., 1977, 1979.

19. Harold OrTON, Eugen DiETH, Survey of English Dialects, Leeds, 1962-71. 


\section{Les structures périphrastiques}

\section{$B e+V$-ing}

Une des caractéristiques du dialecte anglo-gallois est son usage particulier de la forme $B e+V$-ing. Selon Thomas ${ }^{20}$, cette structure est caractéristique des locuteurs bilingues ou issus de la première génération de locuteurs anglophones monolingues. L'usage de cette forme est principalement observé dans les zones bilingues du nord et de l'ouest du pays de Galles car ces dernières furent anglicisées plus tardivement ${ }^{21}$. Il faut également prendre en compte le fait que l'influence du gallois est croissante d'Est en Ouest le long de ce continuum ${ }^{22}$.

La valeur aspectuelle des formes $B e+V$-ing dans le dialecte anglogallois diffère très nettement de l'anglais standard puisque ces constructions servent à décrire des habitudes dans le présent ou le passé :

«He's going to the cinema every week ${ }^{23}$.»

[« Il va au cinéma chaque semaine.»]

Cette valeur aspectuelle de la forme be+Ving est influencée par la construction galloise « $Y+$ Bod (be) + Sujet $+Y n+$ Nom verbal» employée pour exprimer une habitude dans le présent ${ }^{24}$.

«Mae Sandra yngweithio mewn ysgol» [«Sandra travaille dans une école. »]

Littéralement : Sandra is in work in a school $\rightarrow$ Sandra works in a school.

Nous observons une construction similaire à l'imparfait qui sert à nouveau à décrire une habitude dans le passé $:$ «Bod (être) + Sujet $+Y n$ (en/dans) + Nom verbal ».

20. Alan R. THOMAs, «Welsh English: a grammatical conspectus », dans W. Viereck (dir.), Focus on England and Wales, Amsterdam, Benjamins, 1985, p. 213-221.

21. D. PARRY, op. cit., 1979, p. 148. Robert J. PENHALLURICK, «The grammar of Northern Welsh English: progressive verb phrases ", dans J. Klemola, M. Kytö, M. Rissanen (dir.), Speech Past and Present: Studies in English Dialectology in Memory of Ossi Ihalainen, Frankfurt am Main, Lang, 1996, p. 309.

22. N. COUPLAND et A. ThOMAS, op. cit., p. 5.

23. Exemple extrait d'Alan R. THOmAS, «English in Wales », dans R. Burchfield (dir.), The Cambridge History of the English Language, Vol. V: English and Britain and Overseas: Origins and Development, Cambridge, CUP, 1994, p. 94-147.

24. D. PARrY, op. cit., 1979, p. 188. 


\section{Do périphrastique}

La forme progressive be $+V$-ing n'est cependant pas la seule manière d'exprimer l'habitude dans le présent ou le passé. En effet, l'extrême sud et le sud-est du pays de Galles (régions les plus anciennement anglicisées) privilégient l'emploi du do périphrastique pour exprimer cette notion d'habitude :

«She do wear the trousers ${ }^{25}$. » [« Elle porte la culotte. »]

«You do have 'em by the hundred ${ }^{26}$. " [ "Tu les trouves par centaines. »]

Contrairement à la construction emphatique, le do périphrastique est non accentué et demeure non fléchi. Ainsi, la forme $B e+V i n g$ et le do périphrastique sont distribués de manière complémentaire sur le territoire gallois. Les formes en be sont présentes dans les régions bilingues du nord et de l'ouest du pays alors que les constructions en do sont majoritairement répertoriées dans le sud et l'est du pays de Galles ${ }^{27}$. Selon Parry ${ }^{28}$ et Penhallurick ${ }^{29}$, les deux formes sont en compétition dans la province du Glamorgan, région limitrophe la vallée de la Rhondda.

La distribution de cette forme périphrastique serait influencée par le superstrat des dialectes du sud-ouest de l'Angleterre ${ }^{30}$. En effet, l'anglicisation du pays de Galles s'est d'abord produite dans le Sud et l'Est. L'intérieur du pays s'est anglicisé bien plus tard, à partir de la fin du XIX siècle. L'anglicisation a donc eu lieu à travers des médiums différents tels que l'éducation et les médias. Par conséquent, le sud et l'est du pays de Galles ont des caractéristiques linguistiques qui leur sont propres. L'emploi du do périphrastique est caractéristique des vernaculaires du sud-ouest de l'Angleterre, où ces formes furent

25. D. PARRY, op. cit., 1977, 1979.

26. Ibid.

27. À l'exception du Gwent et du Gowver qui dérogent à cette règle. Ces régions ayant été anglicisées dès le Moyen Âge, l'influence du substrat gallois est donc très minime voire inexistante.

28. D. PARRY, op. cit., 1977, p. 161.

29. Rob J. PenHallurick, The Anglo-Welsh Dialects of North Wales: A Survey of Conservative Rural Spoken English in the Counties of Gnynedd and Chwyd, Frankfurt am main, Bern, New York, Paris, Peter Lang, 1991, p. 187-193.

30. R. Penhallurick, op. cit., 1996, p. 33 ; A. R. Thomas, op. cit., 1985, p. 215. 
attestées dès le XIII ${ }^{e}$ siècle ${ }^{31}$. Cette construction était employée en anglais standard jusqu'au XVII siècle, avant de disparaître de l'anglais littéraire. L'usage de cette construction perdura cependant dans les dialectes du sud-ouest de l'Angleterre.

Son origine fait l'objet de nombreux débats, certains défendant l'hypothèse d'un substrat brittonique ${ }^{32}$ d'autres prônant l'hypothèse inverse, celle d'un superstrat anglais ${ }^{33}$. Concernant le dialecte anglogallois, la distribution régionale du do périphrastique favorise l'hypothèse d'un superstrat en provenance du sud-ouest de l'Angleterre ${ }^{34}$. En effet, l'anglicisation du pays de Galles fut en grande partie engendrée par des mouvements de populations originaires du sud-ouest de l'Angleterre. L'hypothèse d'un substrat gallois ne peut cependant être complètement écartée puisqu'une structure similaire existait en moyen gallois. L'emploi de la forme progressive dans cette région semble néanmoins constituer un substrat plus récent car le développement de cette construction en anglo-gallois paraît aller de pair avec le développement du bilinguisme dans les régions concernées ${ }^{35}$.

\section{$B e+V$-ing et do périphrastique dans Cwmardy}

$B e+V$-ing

Dans l'œuvre, la forme $B e+V$-ing est construite de deux manières : soit à l'aide de l'auxiliaire be fléchi, soit à l'aide de be invariant :

«Is your poor little head paining? (Big Jim, chapitre 14)

[《Est-ce que tu as mal à la tête? »]

"You be laughing now.» (Shane, chapitre 12)

[«Tu rigoles maintenant.»]

31. David Denison, English Historical Syntax: Verbal Constructions, London, New York, Longman, 1993, p. 264.

32. Patricia Poussa, «A Contact Universal Origin of Periphrastic DO with Special Consideration of Old English - Celtic Contact», dans S. Adamson (dir.), The 5th International Conference on English Historical Linguistics, Amsterdam, John Benjamins, 1990, p. 407-434.

33. Alvar ElLEGARD, «The Auxiliary Do: The Establishment and Regulation of its Use », Gothenburgh Studies 2, Stockholm, Almqvist and Wiskell, 1953.

34. Heli PITKÄNEN, Welsh English Syntax: Contact and Variation, Thèse doctorale, University of Joensuu, 2005, p. 49.

35. Ibid. p. 226. 
Nous observons également des formes fléchies non standard, caractéristique observée dans la plupart des variétés de l'anglais :

«You was suckling on my breast.» (Shane, chapitre 4)

[«Tu prenais le sein.»]

Les formes $\mathrm{Be}+V$-ing construites à l'aide de be non fléchi sont assez fréquentes dans le roman puisqu'elles représentent $61,4 \%$ des instances pour le personnage de Big Jim et $69 \%$ pour Shane. D'un point de vue sociolinguistique, la forte présence de formes invariantes semble surprenante. En effet, George ${ }^{36}$ ne répertorie qu'une seule instance de ce type ("It be bitching into that shaft»). Penhallurick ${ }^{37}$ et Parry $^{38}$ ne mentionnent aucune structure de ce type. Les enregistrements étudiés à partir du corpus de la bibliothèque des mineurs ne recensent que deux formes progressives invariantes. Par conséquent, Lewis Jones semble sur-employer cette variable. À nouveau, nous retrouvons la même dichotomie que celle précédemment énoncée entre la première et la deuxième partie de l'œuvre. Indéniablement, ces formes existaient à l'époque mais une telle différence dans les fréquences d'emploi laisse perplexe, tant elle diffère des études sociolinguistiques menées dans la région. De plus, la structure progressive $a+V$-ing (ex. a-cutting, a-running), également répertoriée dans le vernaculaire anglo-gallois (et également influencée par l'anglais du sud-ouest en Angleterre), est absente du roman.

Parmi les discours des trois personnages principaux, 124 formes progressives sont employées de manière standard, contre seulement trois servant à marquer l'habitude dans le présent. Lewis Jones emploie donc la forme Be+Ving principalement de manière standard dans l'œuvre :

"I am not getting half the food I was having when dad was home ${ }^{39}$. " (Len, chapitre 17)

["Je n’ai pas la moitié de la nourriture que j'avais quand ton père était à la maison. »]

36. C. GEORGE, op. cit., p. 242.

37. R. J. Penhallurick, op. cit., 1991 et 1996.

38. D. PARRY, op. cit., 1977.

39. [«Je n’ai pas la moitié de la nourriture que j’avais quand ton père était à la maison. »] 
Ces données contrastent fortement avec celles présentées par Pitkänen ${ }^{40}$ qui observe des fréquences d'occurrence bien plus élevées de la forme $B e+V$ ing en contextes itératifs, de l'ordre de $20 \%$ en moyenne. Par conséquent, les idiolectes des personnages principaux semblent être bien loin de la réalité linguistique de l'époque. De plus, l'une des caractéristiques de ce dialecte est l'emploi des formes Be $+V$-ing avec des verbes statifs. Or seulement une instance de ce type est répertoriée dans le corpus (exemple précédent). Une utilisation plus fréquente de cette construction aurait indéniablement rendu la représentation du vernaculaire local plus idiomatique.

\section{Do périphrastique}

184 formes périphrastiques sont recensées dans le roman contre seulement 18 formes emphatiques, ce qui laisse entendre que Lewis Jones a semblé privilégier cette variable plutôt que la structure be + $V$-ing:

«when I did use to walk the fields of the North [...]. » (Big Jim, chapitre 1)

[« quand je me promenais dans les champs dans le Nord »].

Dans les vernaculaires anglo-gallois et du sud-ouest de l'Angleterre, le do périphrastique sert à exprimer l'habitude dans le passé ou le présent. Or, il apparaît très nettement que cette valeur est loin d'être associée aux do périphrastiques présentes dans l'œuvre:

\begin{tabular}{lll} 
& $\begin{array}{l}\text { Do/did : référence à } \\
\text { un procès habituel }\end{array}$ & $\begin{array}{l}\text { Do/did: référence à } \\
\text { un procès ponctuel }\end{array}$ \\
\hline Big Jim & $32(33 \%)$ & $65(67 \%)$ \\
Shane & $21(29 \%)$ & $52(72 \%)$ \\
Len & $7(50 \%)$ & $7(50 \%)$ \\
\hline
\end{tabular}

Il est fort surprenant que le do périphrastique fasse référence à des procès ponctuels. En effet, Ihalainen ${ }^{41}$ ne répertorie aucune instance dans lesquelles le do périphrastique fait référence à une action

40 H. PitKänen, op. cit., p. 252.

41. Ossi Ihalainen, «Periphrastic Do in Affirmative Sentences in the Dialect of East Somerset », Neuphilologische Mitteilungen, t. 77, 1976, p. 609-622. 
spécifique dans le passé dans le vernaculaire du Somerset ${ }^{42}$. Or nous recensons plusieurs exemples dans lesquels l'auxiliaire did fait référence à un procès ponctuel, sans pour autant avoir une valeur emphatique :

«Last time you put these on, was when we did burry our Jane bach» (Shane, chapitre 6) [«La dernière fois que tu les as portés c'est quand on a enterré notre petite Jane. »]

Le do périphrastique est parfois même employé dans des contextes irréels, valeur qui n’est pas recensée par les spécialistes de ce vernaculaire :

«I was bound to laugh, mun, if Dai did kill me for it.» (Big Jim, chapitre 7) ["J'étais obligé de rire, même si Dai m’avait tué pour ça.»]

Il y a donc, à nouveau, une inadéquation entre la forme employée et la valeur aspectuelle qui lui est normalement associée.

\section{Conclusion}

La représentation du vernaculaire anglo-gallois de Lewis Jones, qui peut sembler idiomatique au premier abord, se révèle peu plausible après l'analyse des valeurs aspectuelles des formes non standard présentes dans l'œuvre.

Il y a non seulement des disparités dans les contextes d'emploi de ces variables entre les personnages mais nous observons également une standardisation soudaine de l'idiolecte des personnages principaux, standardisation qui s'opère dès le chapitre 9. Ce changement est d'autant plus inconsistant qu'il n'est justifié par aucun laps de temps qui permettrait de rendre compte de cette transformation.

Bien que Lewis Jones eût connaissance des variations non standard caractéristiques du vernaculaire anglo-gallois, il semble maîtriser partiellement les conditions d'emploi de ces dernières. La représentation qu'il fait du vernaculaire local s'apparente plus à de l'hyperdialectalisme ${ }^{43}$ qu'à une reproduction fidèle du parler de l'époque. À travers

42. Le Somerset est un comté situé dans le sud-ouest de l'Angleterre. Étant donné le lien fort qui unit ces deux dialectes quant à l'emploi de la forme périphrastique, cette conclusion offre un élément de comparaison très précieux.

43. P. TRudgill, op. cit., p. 60. 
de tels choix linguistiques, Lewis Jones parait uniquement privilégier l'existence de deux entités culturelles et sociales bien distinctes : les mineurs de la Rhondda opposés au patronat et au capitalisme d'état. Cette dichotomie n'est cependant clairement manifeste que dans la première partie du roman. La standardisation du discours des personnages dans la deuxième partie de l'œuvre pourrait exprimer de manière implicite le fait que Jones considère la langue anglaise, et principalement la langue standard, comme l'arme discursive la plus à même de défendre la cause des mineurs.

\section{Bibliographie}

AwBerRy Gwen, «The English language in Wales», dans H.C.L. Tristam (dir.), The Celtic Englishes, Heidelberg, Winter, 1997, p. 86-89.

AwBerry G., "Welsh », Language in the British Isles, P. Trudgill (dir.), Cambridge, Cambridge University Press, 1984, p. 259-256.

BALL Martin J., FIFE James, The Celtic Language, London and New York, Routledge, 1993.

BeLLIN Wynford, "Welsh and English in Wales », dans P. Trudgill (dir.), Language in the British Isles, Cambridge, Cambridge University Press, 1984, p. 449-477.

BOrSLey Robert D., RoberTs Ian, The Syntax of the Celtic Languages: A Comparative Perspective, Cambridge, Cambridge University Press, 1996.

CLIFFE Charles F., The Book of South Wales, London, 1847.

Coupland Nikolas, Dialect in Use, Sociolinguistic Variation in Cardiff English, Cardiff, University of Wales, 1988.

Egan David, South Wales Miners: A History of the South Wales Mining Valleys 1840-1980, Llandysul, Gomer Press, 1987.

Filpulla Markku, Klemola Juhani, PitKÄNen Heli (dir.), The Celtic Roots of English, University of Joensuu, 2002.

GaCHELIN Jean-Marc, "The Progressive and Habitual Aspects in Non-

Standard Englishes », dans E. Schneider (dir.), Englishes Around the World,

Vol.1: General Studies, British Isles, North America, Amsterdam, Philadelphia, Benjamins, 1997, p. 33-46.

Hopkins Kenneth S., Rhondda Valley History, Ferndale, 1975. 
Ihalainen Ossi, «On Grammatical Diffusion in Somerset Folk Speech », dans P. Trudgill, J. K. Chambers (dir.), Dialects of English: Studies in Grammatical Variation, London, Longman, 1991, p. 290-302.

JONES Ieuan G., «Language and Community in Nineteenth Century Wales », dans K. S. Hopkins (dir.), Rhondda Past and Future, Ferndale, 1974.

Jones Lewis, Cwmardy, We live, Cardigan, Parthian Library of Wales, 2006.

Jones Morris, Thomas Allan R., The Welsh Language: Studies in its Syntax and Semantics, Cardiff, University of Wales Press for the Schools Council, 1977.

Klemola Juhani, KYTÖ Merja, Rissanen Matti (dir.), Speech Past and Present: Studies in Dialectology in memory of Ossi Ihalainen, Frankfurt am Main, Peter Lang, 1996.

Klemola J., « Periphrastic Do in South-Western Dialects of British English: a Reassessment », Dialk.tologia et Geolinguistica 2, 1994, p. 33-51.

LE CORRE Gaëlle, Cwmardy et le dialecte anglo-gallois : étude de la copule be et de la forme be + -ing, mémoire de Master 2 : anglais, Brest, UBO, 2007.

LEWIS Ceri, "Welsh Language », dans K.S. Hopkins (dir.), Rhondda Past and Future, Ferndale, 1974.

MaCAulay Ronald K. S., Language, Social Class and Education: A Glasgow Study, Edinburgh, Edinburgh University Press, 1977.

PArry David R., "Anglo-Welsh Dialects in South East Wales Patterns in the Folk Speech of the British Isles », dans M. F. Wakelin (dir.), Patterns in the Folk Speech of the British Isles, London, 1972, p. 140-163.

PARRY D. R., «A Grammar and Glossary of Conservative Anglo-Welsh Dialects of Rural Wales », NATCECT. Occasional Publications, t. 8, University of Sheffield, 1999.

Penhallurick Robert J., « Welsh Language: A National language? », Dialectologia and Geolinguistica, 1993, p. 28-46.

Penhallurick R. J., Debating Dialect: Essays on the Philosophy of Dialect Study, Cardiff, University of Wales Press, 2000.

Penhallurick R. J., «Welsh English: Phonology », dans E. W. Schneider, B. Kortmann (dir.), A Handbook of Varieties of English: Volume 1: Phonology, Berlin, New York, Mouton de Gruyter, 2004.

Smith Dai, «Leaders and Led», dans K.S. Hopkins (dir.), Rhondda Past and Future, Ferndale, 1974.

Smith D., Lewis Jones, Cardiff, University of Wales Press, 1982.

TrudgILL Peter, Language in the British Isles, Cambridge, London, New York, New Rochelle, Melbourne, Sidney, Cambridge University Press, 1984.

VIERECK Wolfgang, Varieties of English the World: Focus on England and Wales, Amsterdam, Philadelphia, John Benjamin Publishing Company, 1985. 
VIsSER Gerard. J., «Celtic Influence in English », Neophilologus, t. 39, 1955, p. 276-293.

Wakelin Martin F. (dir.), Pattern in the Folk speech of the British Isles, London, The Athlone Press of the University of London, 1972.

Wakelin M.F., The South West of England, Amsterdam, Philadelphia, John Benjamins, 1986.

WrIGHT Joseph, The English Dialect Dictionary, 6 volumes, Oxford, 1898-1905. Wright J., The English Dialect Grammar, Oxford, 1905.

WRIGHT Peter, "Coal Mining language: A Recent Investigation", dans M. F. Wakelin (dir.), Patterns in the Folk Speech of the British Isles, London, Athlone, 1972, p. 32-49. 